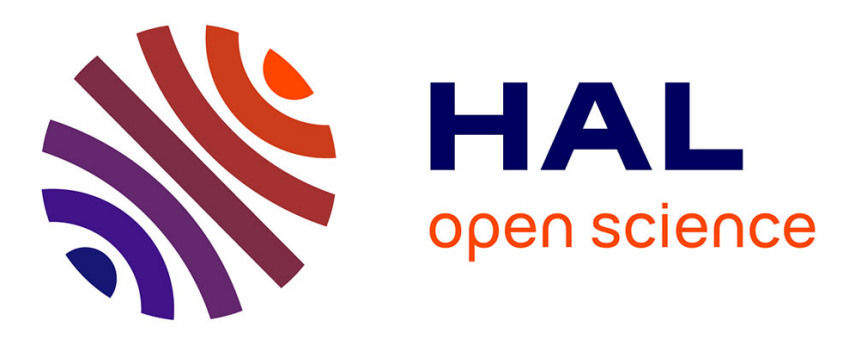

\title{
Mechanically regularized FE DIC for heterogeneous materials
}

Robin Naylor, François Hild, C. Fagiano, M. Hirsekorn, Y. Renollet, B. Tranquart, Emmanuel Baranger

\section{To cite this version:}

Robin Naylor, François Hild, C. Fagiano, M. Hirsekorn, Y. Renollet, et al.. Mechanically regularized FE DIC for heterogeneous materials. Experimental Mechanics, 2019, 59 (8), pp.1159-1170. $10.1007 / \mathrm{s} 11340-019-00529-9$. hal-02156727

\section{HAL Id: hal-02156727 https://hal.science/hal-02156727}

Submitted on 14 Jun 2019

HAL is a multi-disciplinary open access archive for the deposit and dissemination of scientific research documents, whether they are published or not. The documents may come from teaching and research institutions in France or abroad, or from public or private research centers.
L'archive ouverte pluridisciplinaire HAL, est destinée au dépôt et à la diffusion de documents scientifiques de niveau recherche, publiés ou non, émanant des établissements d'enseignement et de recherche français ou étrangers, des laboratoires publics ou privés. 
Experimental Mechanics manuscript No.

(will be inserted by the editor)

\title{
Mechanically regularized FE DIC for heterogeneous materials
}

\author{
R. Naylor · F. Hild • C. Fagiano • M. Hirsekorn • \\ Y. Renollet • B. Tranquart • E. Baranger
}

Received: date / Accepted: date

\begin{abstract}
In situ tensile tests in a scanning electron microscope (SEM) have been conducted on a 8layer 5-harness satin carbon fibre and epoxy matrix composite to observe the first stages of damage at the scale of fibres and matrix. A speckle pattern based on a suspension of alumina particles was applied onto the surface of the specimen to facilitate the use of digital image correlation (DIC). Local and finite element (FE) DIC are compared on pictures acquired during the tensile tests, with and without a speckle pattern. FE DIC with mechanical regularization was found to be the only approach able to measure displacement fields at a fine enough resolution in both cases. This method, initially created for homogeneous materials, was then adapted to heterogeneous materials. First, a microstructure consistent mesh was created and used for correlation purposes. Second, the difference between the mechanical properties of the constituents is taken into account in the mechanical regularization. Last, the accuracy of the method is analysed. The adaptation presented herein was proved to be able to measure displacement fields in the matrix between fibres with an error of $10 \mathrm{~nm}$ (a fifth of a pixel) and to detect the initiation of the first damage mechanisms by means of the mechanical residuals.
\end{abstract}

Keywords Digital Image Correlation, Mechanical regularization, Textile composites, In-situ mechanical test, Microscopic failure

R. Naylor, C. Fagiano, M. Hirsekorn, Y. Renollet

Onera,

29 avenue de la Division Leclerc F-92322 Chatillon CEDEX (FRANCE)

E-mail: robin.naylor@onera.fr

R. Naylor, F. Hild, E. Baranger

LMT (ENS Paris-Saclay/CNRS/Univ. Paris-Saclay),

61 avenue du Président Wilson, F-94235 Cachan CEDEX (FRANCE)

R. Naylor, B. Tranquart

SAFRAN Composites,

31 avenue de la gare, F-91760 Itteville CEDEX (FRANCE) 


\section{Introduction}

Carbon fibre and epoxy matrix woven composites are widely used nowadays in the aeronautical industry even for primary structural components [1]. These materials have a multi-scale nature and three characteristic scales can be defined. First, the macroscopic scale corresponds to that of the part or the structure of interest. Second, the scale of the architecture or woven pattern will be referred to as the mesoscopic scale. Finally, the microscopic scale is that of fibres and matrix. In the case of the composite under investigation, the first damage mechanisms occur at the microscopic scale. It consists in fibre/matrix debonding and matrix cracking [2]. They coalesce and lead to mesoscopic scale damage in the form of transverse cracks inside yarns and delamination along the interfaces between yarns [2]. Last, the increase in density of mesoscopic scale damage and fibre breakage leads to failure of the structure [2-8]. This damage scenario can be found in a wide range of composites. The constituents (including their interfaces) have a strong influence on damage onset and growth at the microscopic scale and thus on the failure of the structure [9]. This is especially true in the case of fatigue loadings where repeated loading can cause the growth of microscopic scale damage even at load levels lower than those necessary to generate mesoscopic scale damage.

In order to be predictive for the onset of damage with numerical models, accurate means of characterisation are required. However, the characterisation of the first stage of damage at the microscopic scale is difficult and rarely performed. The main reasons are: (i) the difficulty to measure the properties of the constituents and of their interfaces [10], and (ii) the difficulty to measure displacement fields at the microscopic scale and to define the damage scenario. Concerning this second point, the challenge is due to the smallness of the displacements that have to be measured. At this scale, the size of the damage mechanisms is about a few nanometres [2] and thus of the same magnitude as the displacement. To be able to observe them, experiments are carried out with a scanning electron microscope (SEM) to obtain grey level pictures of the sample surface. This observation can be performed either post-mortem [11,12], at the end of an interrupted test $[13,14]$ or even in situ in the SEM $[15,16]$.

From these images, a widely used technique to measure displacement fields is digital image correlation (DIC) [17-19]. This method is based on the grey levels of the picture and assumes that they are maintained between the original image and those after deformation. However, some issues can arise from the SEM acquisition of the digital pictures of composite materials. First, the stability of grey levels is not ensured when acquiring SEM pictures as the current intensity of the electron beam can vary. Second, the material (i.e., the epoxy matrix in the present case) being an electrical insulator, a charging effect can appear and affect the grey levels [20]. Third, in the case of pictures with uniform grey levels, it is necessary to deposit a microscopic speckle pattern on the surface of the sample. The common method used at the macroscopic scale is to spray paint droplets on the surface of the 
sample [21]. However, it is difficult to spray microscopic size droplets and their size is often of the order of several tens of micrometres [22]. To overcome this difficulty, the method used herein creates a speckle pattern from a water-based solution containing ceramic particles [23]. This method presents the advantages to (i) cover the whole surface of the sample contrary to electron-beam lithography methods [24-26], (ii) be easily created contrary to metal loaded polymer layer methods [27,28] and (iii) keep the microstructure of the sample visible contrary to methods based on paints [21, 29]. The ability to keep the microstructure visible is especially useful at the stage of creating a FE mesh of a representative volume element at the microscopic scale to compare experimental observations with numerical simulations.

Two different approaches of DIC can be found in the literature, namely, the local $[17,30,31]$ and the global approaches. The last one can be further decomposed in finite element (FE) approaches [32, 33], which is considered here, and other approaches such as Fourier-based approach [34]. The local approach consists in dividing the whole picture into small subsets and analysing them independently, whereas the FE approach considers the whole picture by sampling the displacement field over a finite element (FE) mesh. The advantage of local approaches is the speed of calculation since many small problems are faster to solve (possibly in parallel) than a larger system. The major advantage of FE-DIC is its natural bridge with numerical simulations. Besides, the use of a finite element mesh to sample the displacement fields allows the use of several methods to improve the resolution by adding other terms to the minimization [35] or by using regularization methods based on a proper generalized decomposition (PGD) [36] or a mechanical equilibrium [37,38]. Furthermore, the FE approach, due to the use of a finite element mesh ensuring continuity of the field, can measure more complex fields and is more robust in the case of noisy images as shown by Hild et al. [39] and by the comparison of the works of Canal et al. [14] with Mortazavi [34], who used the same SEM pictures.

This work proposes a procedure based on FE DIC with mechanical regularization able to capture displacement fields with high resolution at the microscopic scale on heterogeneous materials with a high stiffness ratio, which is the case for carbon fibre polymer resin (CFPR) composites. The first section presents a comparison between two existing correlation approaches, namely, local DIC performed using the commercial code VIC-2D [40], and FE and regularized T3 DIC (i.e., Correli RT3 [38]) in order to test the FE approach with mechanical regularization based on three nodes (T3) elements $[37,41]$. Both methods are applied to the considered material with and without a speckle pattern. The adaptation of the FE DIC with mechanical regularization to the measurement of high resolution displacement fields for heterogeneous materials with a high Young's modulus ratio is then introduced. 


\section{Comparison between the local and FE approaches to measure microscale}

\section{displacements}

\subsection{Digital Image Correlation method}

Two different approaches exist for DIC but for both approaches the conservation of the grey level between the reference picture, $f$, and the picture after deformation, $g$, is assumed at any pixel, $\boldsymbol{x}$, for a displacement vector, $\boldsymbol{u}: f(\boldsymbol{x})=g(\boldsymbol{x}+\boldsymbol{u}(\boldsymbol{x}))$ [42].

The correlation procedure aims, for instance, at minimizing the sum of squared differences over a domain, $\Omega$

$$
\Phi_{o}^{2}=\sum_{\Omega}[f(\boldsymbol{x})-g(\boldsymbol{x}+\boldsymbol{u}(\boldsymbol{x}))]^{2}
$$

One of the main differences between both approaches lies in the choice of the domain, $\Omega$. On one hand, the local approach defines numerous small and independent $\Omega$ domains over the picture and finds independent displacements for each of them through Equation (1).

The FE approach defines $\Omega$ as the whole picture through a finite element mesh where the nodal displacements are the components of the displacement vector, $\boldsymbol{u}$, which are to be determined. Minimizing Equation (1) is equivalent to solving a sequence of linear systems

$$
\underline{M} \cdot \delta u=b
$$

where $\underline{M}$ is the Hessian whose terms are the products of the shape functions of the finite element mesh by the gradient of the initial image, $\boldsymbol{b}$ the residual vector, which is a function of the difference between the initial picture and the corrected deformed picture [39], and $\boldsymbol{\delta} \boldsymbol{u}$ the corrections to the current estimate of the nodal displacements. Due to the continuity of the displacement field, the FE approach can measure more complex fields and could be more robust in the case of noisy images [39].

This global correlation problem is ill-posed and many ways have been proposed to transform it into a well-posed problem [43]. The regularization used herein is based on a penalty term written in terms of the equilibrium gap which consists in a minimization of the norm of the internal force residuals [38]. Since it is assumed that there are no external forces, the nodal forces $\boldsymbol{f}_{\boldsymbol{r}}$ should vanish

$$
\underline{K} \cdot u=f_{r}
$$

where $\underline{\boldsymbol{K}}$ is the rectangular stiffness matrix restricted to the inner nodes. It is computed by assembling in a FE sense the elementary stiffness matrices that account for the different elastic properties in the matrix and in the fibres. Plane stress conditions were used. Consequently, the equilibrium gap is 
defined as the L2-norm of the force residuals, or equivalently

$$
\Phi_{m}^{2}=\boldsymbol{u}^{t} \cdot \underline{\boldsymbol{K}}^{t} \cdot \underline{\boldsymbol{K}} \cdot \boldsymbol{u}
$$

Henceforth, the quantity $\underline{\boldsymbol{K}}^{t} \cdot \underline{\boldsymbol{K}} \cdot \boldsymbol{u}$ is defined as the mechanical residual, which is the work conjugate of the nodal displacement vector.

To minimize the global problem, both functionals are normalized by a trial displacement field in the form of a plane wave $\boldsymbol{v}(\boldsymbol{x})=v_{0} \cdot \exp (i \boldsymbol{k} \cdot \boldsymbol{x})$, where $v_{0}$ is the amplitude and $\boldsymbol{k}$ the wave vector. The normalized functionals then become

$$
\tilde{\Phi}_{m}^{2}=\frac{\Phi_{m}^{2}}{\boldsymbol{v}^{t} \cdot \underline{\boldsymbol{K}}^{t} \cdot \underline{\boldsymbol{K}} \cdot \boldsymbol{v}} \quad, \quad \tilde{\Phi}_{o}^{2}=\frac{\Phi_{o}^{2}}{\boldsymbol{v}^{t} \cdot \underline{\boldsymbol{M}} \cdot \boldsymbol{v}}
$$

The global minimization is performed on the weighted sum of the two normalized functionals [38]

$$
\left(1+w_{m}\right) \cdot \Phi_{t}^{2}=\tilde{\Phi}_{o}^{2}+w_{m} \tilde{\Phi}_{m}^{2}
$$

where $w_{m}$ is the weight associated with the penalization related to the equilibrium gap. The weight $w_{m}$ becomes

$$
w_{m}=2 \pi\left(|\boldsymbol{k}| \ell_{m}\right)^{4}
$$

where $\ell_{m}$, is referred to as the regularization length [38].

It is worth noting that the mechanical regularization is used herein as a guide for the minimization scheme through an iterative process. The registration is initially performed with a high weight of the mechanical part. The displacement fields obtained at convergence (i.e. for the L2-norm of the displacement corrections less than $10^{-5}$ pixels) are then used to initialize a new registration step with less weight on the mechanical part (typically half the previous regularization length). Such relaxation process is conducted until it no longer converges for a maximum number of iterations of 200 .

\subsection{DIC analyses of the considered material}

In order to acquire images, in situ tensile tests were conducted in an SEM. The acquired pictures have a resolution of $46 \mathrm{~nm} /$ pixel. The considered material is an 8-layer 5-harness satin carbon fibre and epoxy matrix composite (Fig. 1a). The sample dimensions are $40 \times 3.8 \times 2 \mathrm{~mm}^{3}$. Its surface is polished to one quarter of micrometre. In order to observe the first stages of damage at the microscopic scale, the acquisition parameters should be chosen with care. First, to observe damage initiation, i.e., fibre/matrix debonding, the resolution must be high enough (about 50nm). Moreover, since the location of the transverse cracks cannot be predicted a priori, a large zone of the composite must be observed (i.e., about $800 \times 300 \mu \mathrm{m}$ ). Then, to prevent the effect of rigid body motions and 
keep the same observed zone in the acquired pictures during the test, high resolution pictures should be captured (about $4000 \times 4000$ pixels). Finally, the electron beam power should be adapted in order to avoid damaging the composite. All these requirements imply other aspects such as the speckle pattern or the DIC analyses to be adapted.

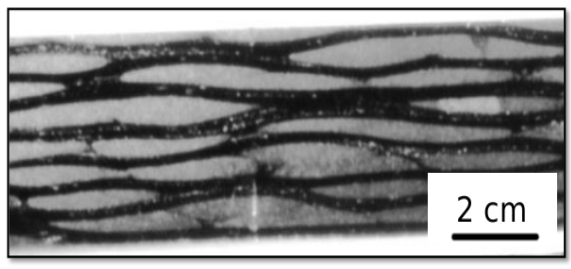

(a)
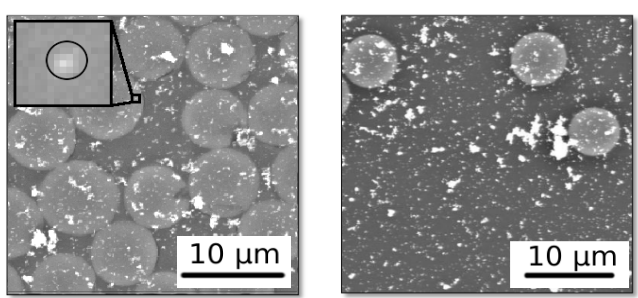

(b)

Fig. 1: (a) Optical picture of the side of the composite sample. (b) (left) speckle pattern on the yarn with a zoom at the pixel level, (right) speckle pattern on a large interyarn matrix pocket.

A speckle pattern was created with a method similar to that developed by Mehdikhani et al. [23] based on a suspension of alumina particles, about $125 \mathrm{~nm}$ in diameter (Fig. 1b) corresponding to 2 to 3 pixels [44]. DIC was applied during a tensile test with a speckle pattern and has confirmed the absence of displacement of each individual particle.

\subsubsection{Comparison between local and FE-DIC with speckle pattern}

For the local approach (Fig. 2(b) and 2(c)), a subset size of 21 pixels gives a displacement field with a good spatial resolution. A subset size of 61 pixels (i.e., half the fibre diameter) is required to measure the displacement field all over the picture (using the default parameters of VIC-2D [40] to select reliable measurements). In the case of FE-DIC with mechanical regularization (Fig. 2(a)), a mesh with an element size of 10 pixels and a regularization length of 128 pixels allows displacement fields to be measured over the whole domain. This comparison shows that, with a speckle pattern, both approaches can measure displacement fields over the surface with a comparable spatial resolution. 


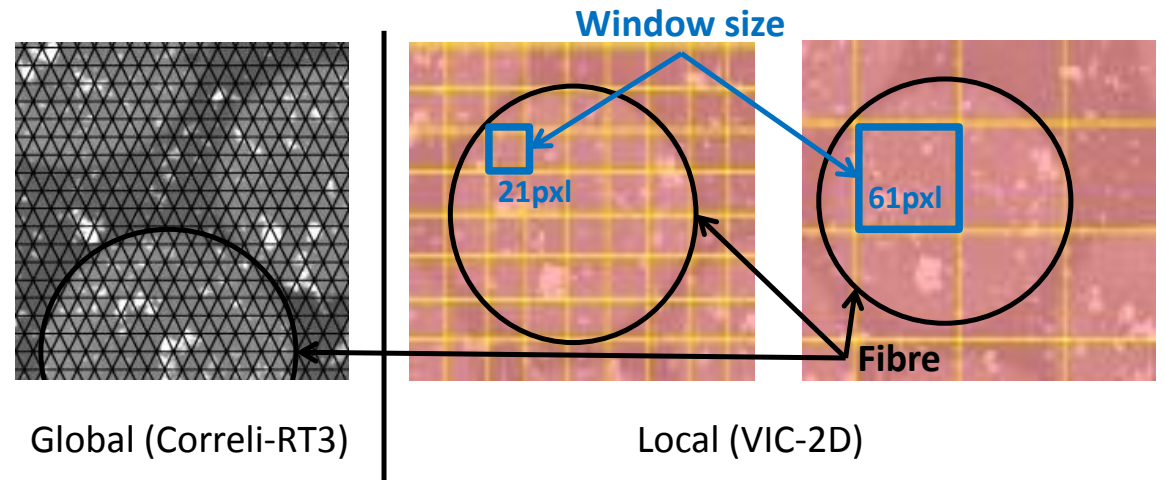

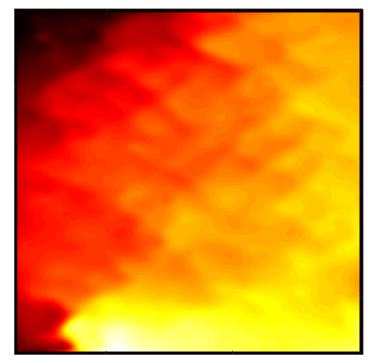

(a)

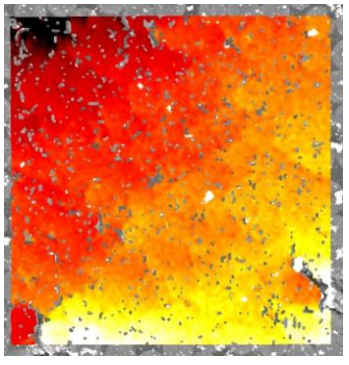

(b)

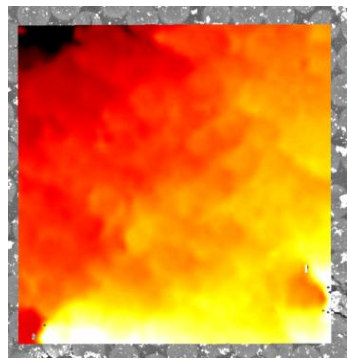

(c)

Fig. 2: Top row. Size of the elements and subsets used for the correlation analyses. Bottom row. The measured horizontal displacement fields (a) FE DIC with 10-pixel elements (and 128-pixel regularization length), (b) local DIC with a subset size of 21 pixels and a step size of 7 pixels, (c) local DIC with a subset size of 61 pixels and a step size of 7 pixels. The dimensions of the field of view are $1500 \times 1500$ pixels. The resolution is $46 \mathrm{~nm} /$ pixel.

\subsubsection{Comparison between local and FE-DIC with no speckle pattern}

The aim is to observe the first stages of damage and to be able to characterise them in terms of strain level or location, for instance. However, using the speckle pattern implies dipping the sample into water whereas such composite materials are sensitive to moisture. One of the main effects is the weakening of the fibre/matrix interface directly linked to the first damage [45, 46]. Thus, there is a need for measuring displacement fields on composite samples without a speckle pattern.

For the local approach, a subset size equal to that of one fibre (i.e., 140 pixels) gives insufficiently resolved displacement fields (Fig. 3(c)) and any smaller subset size does not enable displacements to be measured over the whole region of interest (Fig. 3(b)). The FE approach, with a regularization length of 128 pixels and a mesh with an element size of 10 pixels (Fig. 3(a)), allows displacement fields to be measured over the whole domain (Fig. 4). The SEM images contain many regions with almost uniform gray levels and the displacement fields measured with both methods show that, due to the mechanical regularization, FE-DIC gives more complete measurements than local DIC. 


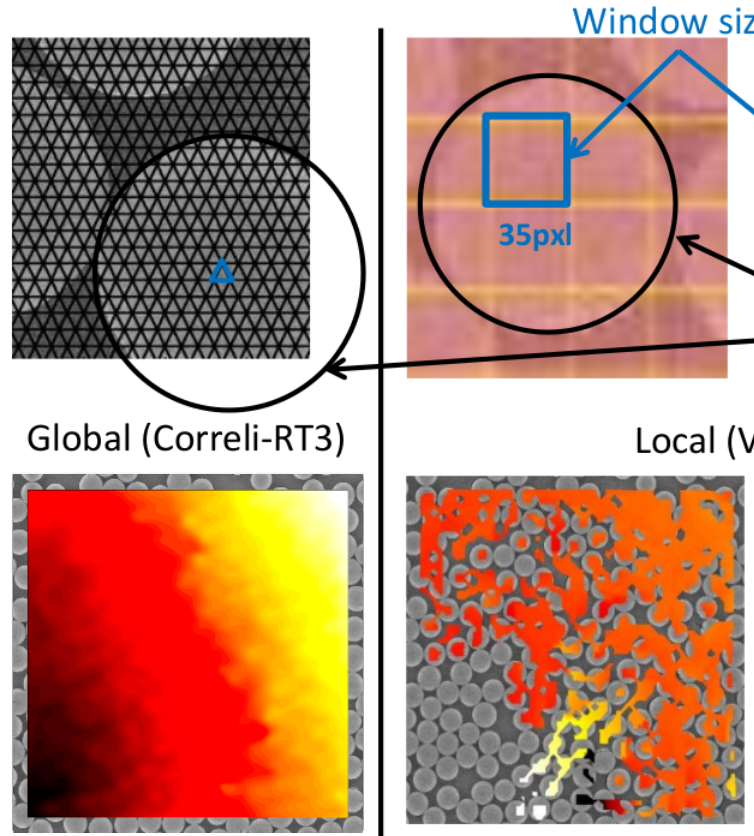

(a) (b)
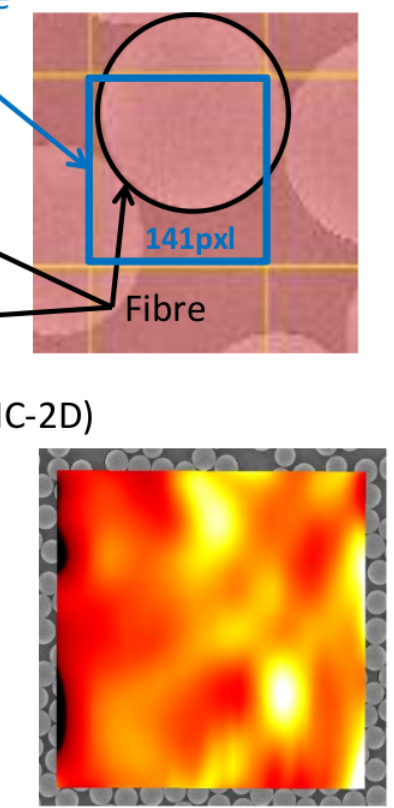

(c)

Fig. 3: Top row. Size of the elements and subsets used for the correlation analyses. Bottom row. The measured horizontal displacement fields (a) FE DIC results with 10-pixel elements (and 128-pixel regularization length), (b) local DIC with a subset size of 35 pixels and a step size of 1 pixel, (c) local DIC with a subset size of 141 pixels and a step size of 1 pixel. The dimensions of the field of view are $2100 \times 2000$ pixels. The resolution is $46 \mathrm{~nm} /$ pixel.

\subsubsection{Choice of method}

For the considered pictures, FE-DIC will be used in the following study. The results presented in the sequel are obtained from the picture shown in Fig. 3(a) and used for the comparison of both methods. The reference image is that of the undeformed sample, and the deformed image is taken at a global tensile stress of around $200 \mathrm{MPa}$. The only exception is in section 3.3 where both reference and "deformed" pictures are acquired for the undeformed state. All the results are presented for a regularization length of 128 pixels as this is the smallest value leading to convergence on the correlation process according to the criteria mentioned in Section 2.1. However, the registration process was initialized with a regularization length of 1024 pixels, which was then gradually decreased (as explained in Ref. [38]). The regularization length of 128 pixels is of the order of the average size of the fibres (120 pixels in diameter).

Figure 4 shows a more detailed result obtained with this method on a sample without a speckle pattern. The mechanical residuals are interpolated from nodal to pixel values using the shape functions of the FE mesh. Another aspect (which is not developed in this paper) justifying the interest of the FE approach, is the possibility of using the same mesh for mechanical simulations. The use of the 
same mesh allows for faster and easier comparisons between numerical simulations and experiments because the same kinematic basis is used in both cases.

However, the use of FE DIC at this scale requires some improvements due to the mechanical residuals all over the fibre/matrix interface (Fig. 4c) preventing its utilisation to detect damage. This phenomenon is due to the presence of some elements lying across the fibre/matrix interfaces; thus, the mechanical regularization procedure considers only one material behaviour for two very different constituents. This is due to the use of a uniform mesh over the considered domain (see top of Fig. 3(a) or Fig. 5a). Moreover, the fields displayed in Figs. 4a and 4b show that their spatial resolution is not sufficient in order to be compared with numerical simulations since it does not capture precisely the interfaces between fibres and matrix. This is due to the assumption of homogeneous elasticity in the regularization process whereas the mechanical behaviour of fibres and matrix are really different $[47,48]$.

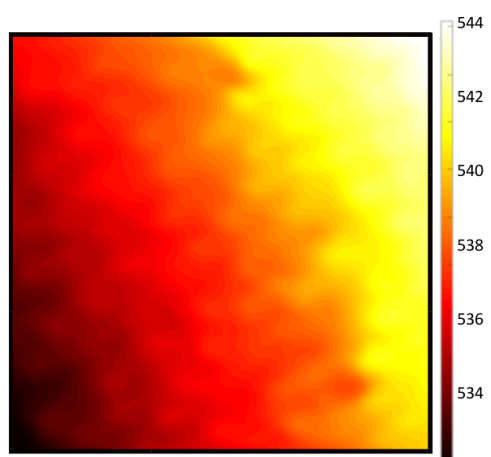

(a)

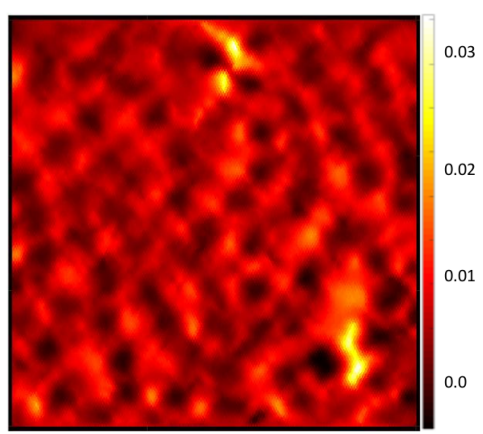

(b)

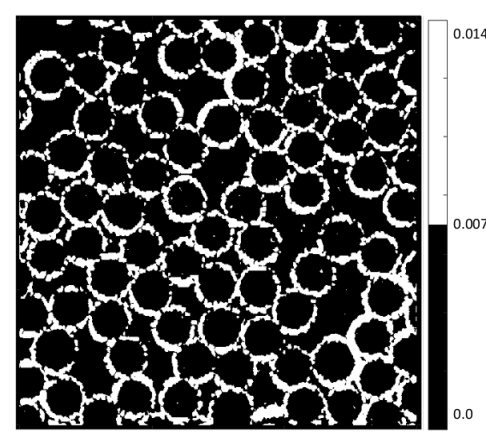

(c)

Fig. 4: (a) Displacement field in the horizontal direction (expressed in pixels, 1 pixel $\equiv 46 \mathrm{~nm}$ ), (b) strain field in the horizontal direction, and (c) mechanical residual (arbitrary unit) for a uniform mesh of 10-pixel elements and a 128-pixel regularization length).

In order to be able to use the mechanical residuals to detect damage, the regularization procedure needs to be extended by taking into account the heterogeneous properties of the material. First, a conformal mesh is required for the correlation analysis. Second, different material parameters will be used for fibres and matrix. Since dipping the sample into a water-based solution to do the patterning can change the way damage initiates at the microscopic scale, the new DIC procedure will be tested on pictures without a speckle pattern (Section 3). As the speckle patterns make the correlation easier, all the new implementations also work in that case. 


\section{FE-DIC on heterogeneous materials}

\subsection{Microstructure-based mesh}

The grey level contrast between fibres and matrix in the picture is sufficient to detect the centre and radius of each fibre through a succession of topological operations. The cross sections of the fibres are assumed to be circular. Nevertheless, the fibre radius can be different from one fibre to another fibre. Since the radii are detected independently, some circles, representing the fibres, can be overlapping (Fig. 5b) and, in this case, a small reduction in both radii is performed. This process allows to switch from a uniform mesh (Fig. 5a) with elements lying across the fibre/matrix interfaces to a mesh consistent with the underlying microstructure of the composite (Fig. 5c).

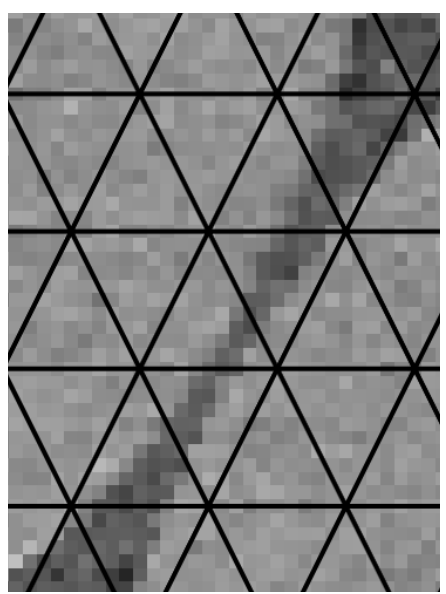

(a)

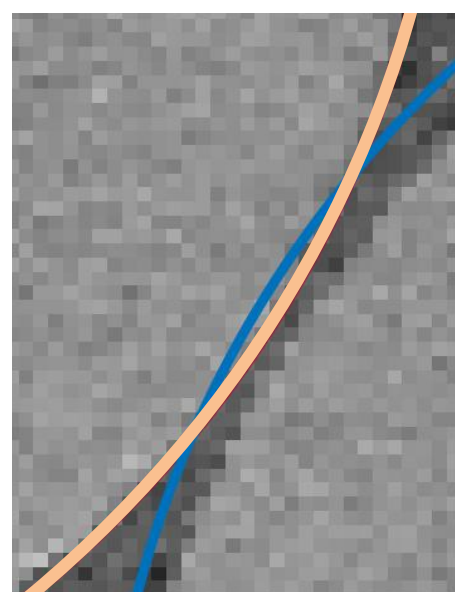

(b)

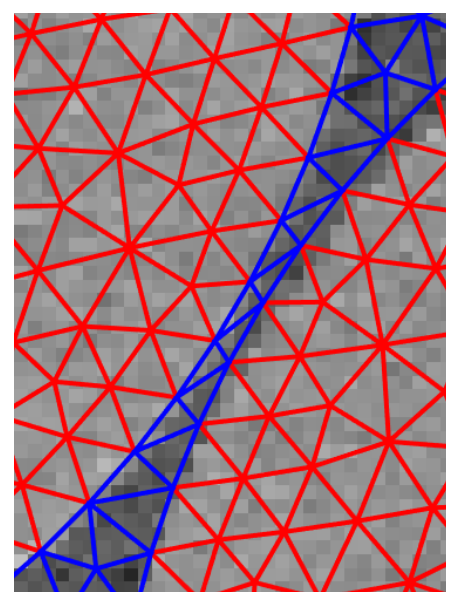

(c)

Fig. 5: (a) Uniform mesh (with 10-pixel elements), (b) overlapping fibre circles and (c) corrected mesh (with a maximal element size of 5 pixels).

The results obtained with this mesh are shown in Fig. 6. As only the positions of the points have changed, there is no real difference between the displacement or strain fields shown in Fig. 4a (resp. 4b) and Fig. 6a (resp. 6b). However, the mechanical residuals do not appear at every fibre/matrix interface anymore but only at some specific places. These residuals are due to fibre/matrix debondings (as can be seen on the zoomed sections of the image shown in Fig. 7(a)) implying a discontinuous displacement field whereas the mechanical model used imposes a continuous displacement field and therefore a large difference at these locations. This is confirmed either visually or with the strain field (Fig. 4b). The use of a microstructure-based mesh allows the detection of the onset of fibre/matrix debonding and allows its growth to be followed. 


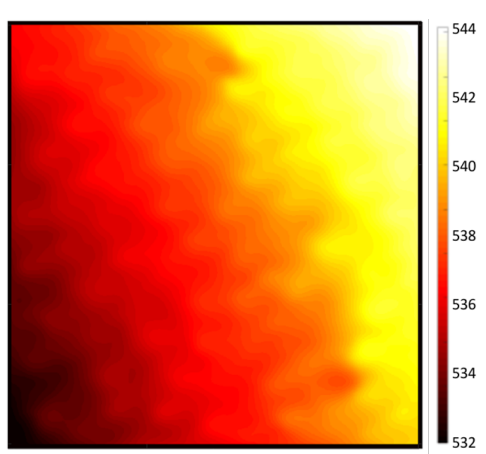

(a)

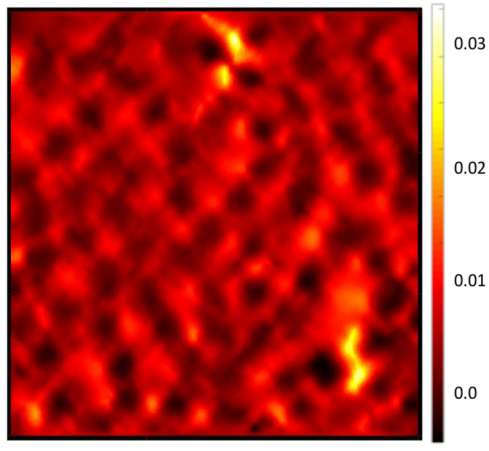

(b)

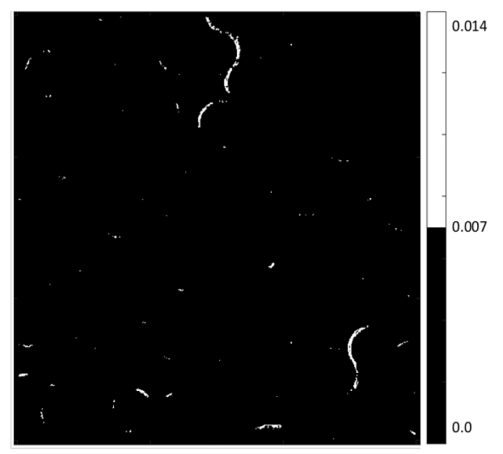

(c)

Fig. 6: (a) Displacement field in the horizontal direction (expressed in pixels, 1 pixel $\equiv 46 \mathrm{~nm}$ ), (b) strain field in the horizontal direction, and (c) mechanical residual (arbitrary unit) for microstructurebased mesh with a maximal element size of 5 pixels and a 128-pixel regularization length.

\subsection{Heterogeneous regularization}

Since the mesh now differentiates the different phases of the composite, it is possible to assign different elastic properties in the regularization method and thus take into account the heterogeneous nature of the composite. For this purpose, different Young's moduli and Poisson's ratios are used for fibres and matrix according to the element location. The stiffness tensor $\underline{\boldsymbol{K}}$ introduced in Section 2.1 is assembled like any FE problem using these properties. A factor of five between the Young's moduli of fibres and matrix is used in this section. It corresponds approximately to the ratio between the properties measured experimentally, between 12 and $15 \mathrm{GPa}$ for the transverse modulus of a high modulus carbone fibre [48-50] and between 2.8 and 3 GPa for the epoxy resin [47,51]. The Poisson's ratios are fixed at 0.3 for both fibres and matrix but its influence is not studied in this article.

The results obtained with this method are shown in Fig. 7. By taking into account the difference between fibres and matrix in terms of mechanical properties, it is now possible to take into account the microstructure of the composite in the displacement fields and thus in the strain fields (Fig. 7). It is observed that the strain levels in the fibres (ca. 0.2\%) are smaller than in most of the matrix (see zoom in Fig. 7e). The only areas where the strains in the matrix are lower than in the fibres are close to fibre/matrix debond zones, which cause unloading of the adjacent matrix. The maximum strain is found at the debonded interfaces between fibres and matrix. However, in comparison with Fig. 6c, the use of different elastic properties for fibres and matrix has a negligible influence on the mechanical residuals. The mechanical maximal value is slightly decreased but the localisation is not changed. This is essentially due to the presence of a fibre/matrix debonding which creates high mechanical residuals hiding mechanical residuals of lower levels. With the aim of detecting the first stages of damage, this is a positive result meaning that whatever the properties used, it does not decrease the ability to detect damage mechanisms. 


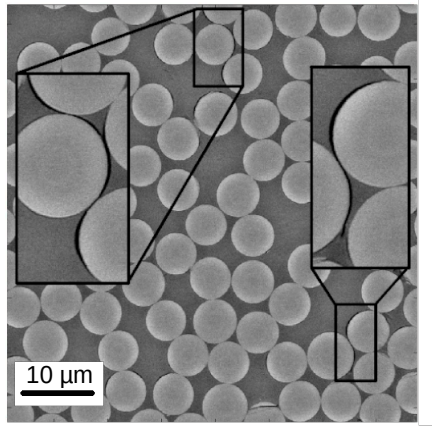

(a)

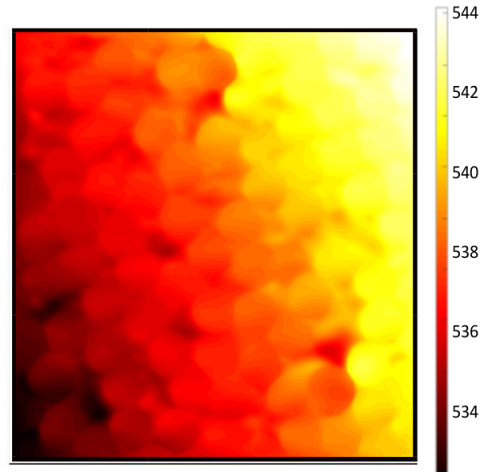

(d)

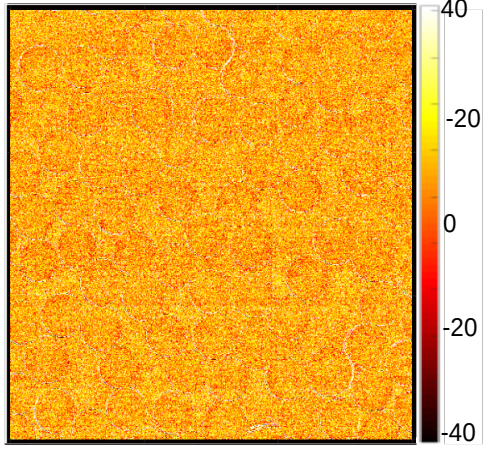

(b)

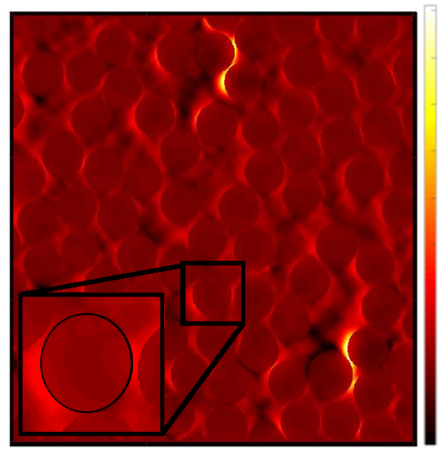

(e)

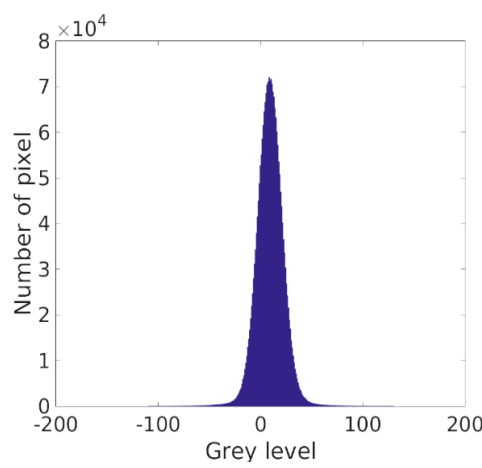

(c)

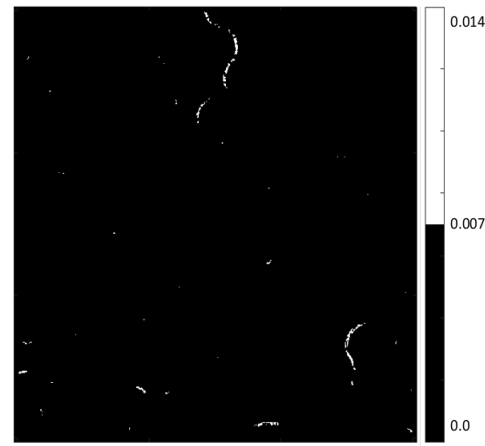

(f)

Fig. 7: (a) Deformed pictures with highlighted fibre/matrix debond areas, (b) grey level residuals, (c) Histogram of the grey level residual, (d) Displacement field in the horizontal direction (expressed in pixels, 1 pixel $\equiv 46 \mathrm{~nm}$ ), (e) strain field in the horizontal direction with a zoom on a fibre with its external surface depicted by a black circle, and (f) mechanical residual (arbitrary unit) with heterogeneous elastic properties and a microstructure-based mesh with a maximal element size of 5 pixels and a 128-pixel regularization length.

The influence of the fibre radius has been analysed and it was found that a 5-pixel reduction, which is quite large considering the very precise positioning of the mesh with respect to the fibre/matrix interfaces as shown in Fig. $5 \mathrm{c}$, only leads to a change of less than 0.1 pixel in the measured displacement fields (which is lower than the measurement uncertainty reported in Section 3.3).

The comparison of the global residuals between the initial method, the use of the microstructurebased mesh and the separation of elastic properties is shown in Fig. 8. As expected, the decrease in the regularization length decreases the optical residual and increases the mechanical one since the mechanical part is less weighted than the optical one. It can also be seen (Fig. 8a) that the use of a microstructure-based mesh does not really change the optical residual since only the positions of the nodes are changed. However, the separation of the elastic properties decreases the optical residual by $2.4 \%$. For the mechanical residual, the proposed improvement induces a reduction of $92 \%$. 


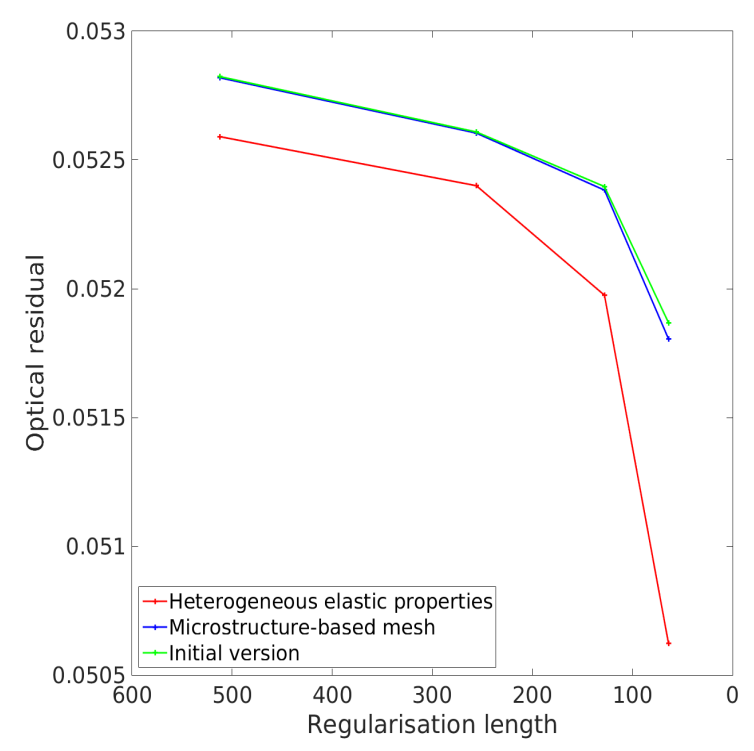

(a)

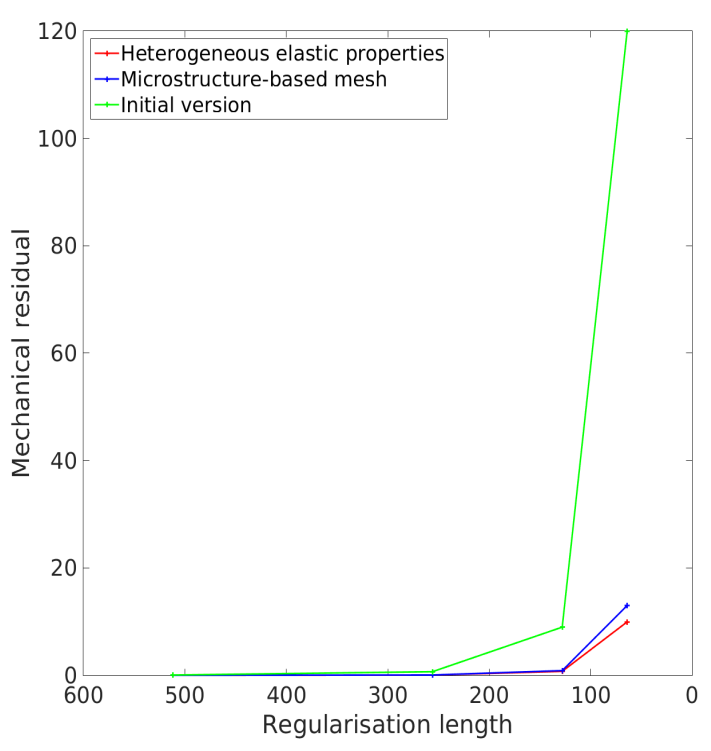

(b)

Fig. 8: Changes in the residuals as a function of the regularization length for the different improvements of the DIC method.

In the same way as the displacement discontinuity at debonded interfaces leads to high mechanical residuals, the fact that the matrix behaviour is not linear elastic may cause higher mechanical residuals in zones with significant inelastic strains. However, the observed values are much lower than on the debonded interfaces even though weight of the mechanical regularization in the matrix for the last converged registration is very small and thus the displacement field in the matrix obtained after the iteration process is not much influenced by the elastic regularization.

\subsection{Uncertainty quantification}

The acquisition of SEM pictures are subject to spatial and drift distortions [52,53]. Models have been developed to correct these distortions [54]. In this paper, the pictures were acquired using a field emission gun SEM known to induce very limited drift compared to conventional tungsten filaments [52] under a short time (i.e., less than one minute by frame). Moreover, the image acquisition was limited to small deviation angles of the electron beam in order to reduce spatial distortion. The aim of this section is to determine the measurement errors including the effect of acquisition artefacts. Two pictures of the same region of interest were thus acquired with a very small rigid body motion of the specimen. The resolution of these pictures was still $46 \mathrm{~nm} /$ pixel and one of them is shown in Fig. 9a. The reported results are obtained with a regularization length of 128 pixels and an elastic contrast of 5 between the two Young's moduli. The pictures being acquired in an SEM, the noise level is quite high in comparison with standard white light cameras. This phenomenon is evidenced 
in the optical residual (Fig. 9d), and thus an increase in measurement uncertainties is expected. The displacement field measured in the horizontal direction (Fig. 9b) has a mean value of 1.03 pixel and a standard deviation of 0.22 pixel, which corresponds to a standard uncertainty of $10 \mathrm{~nm}$ (Fig $9 \mathrm{e})$. In the vertical direction, the mean displacement is -0.57 pixel for a standard deviation of 0.17 pixel, which corresponds to a standard uncertainty of $8 \mathrm{~nm}$ (Fig 9f). This uncertainty is small enough to detect damage since fibre/matrix debonding is expected to be of the order of several tens of nanometres.

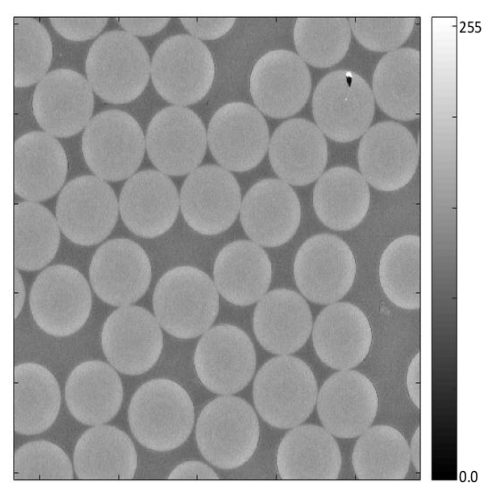

(a)

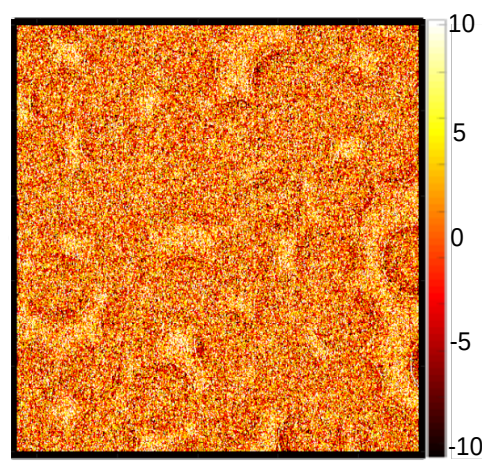

(d)

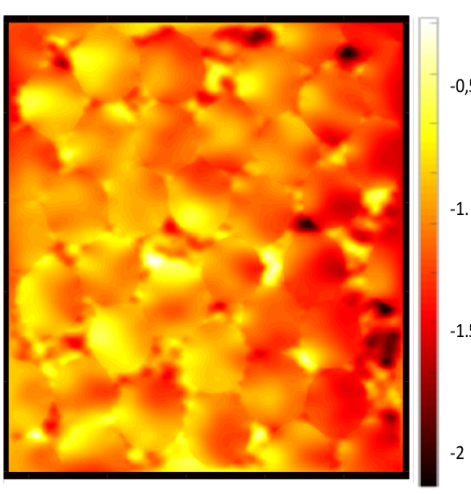

(b)

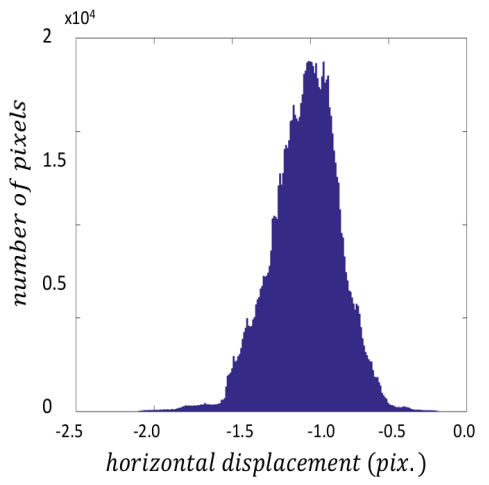

(e)

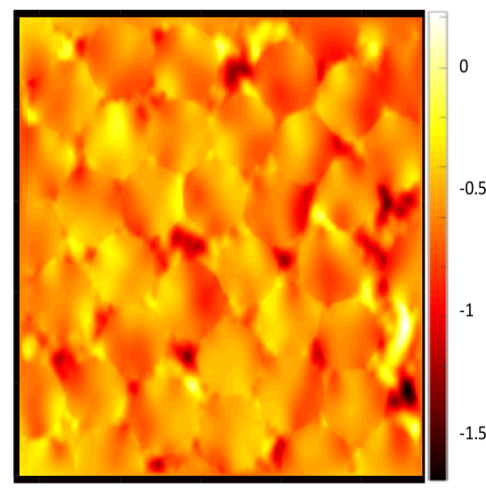

(c)

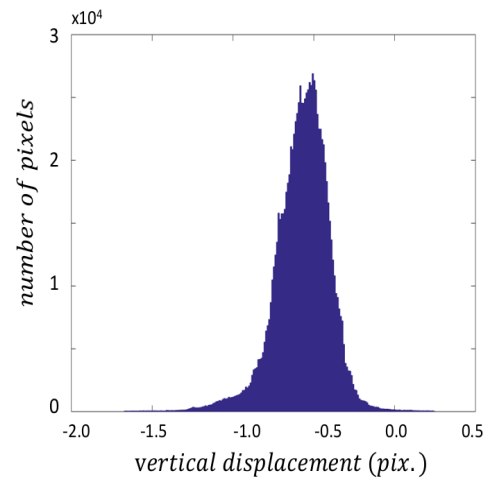

(f)

Fig. 9: (a) Reference picture. Displacement field (expressed in pixels, 1 pixel $\equiv 46 \mathrm{~nm}$ ) along the horizontal (b) and vertical (c) directions. (d) dimensionless grey level residual field. Histogram of the displacements in the horizontal (e) and vertical (f) directions.

\subsection{Influence of the elastic contrast}

This section evaluates the influence of the ratio between the Young's moduli of the two constituents. It is worth noting that the regularization weight $w_{m}$ depends on the elastic properties, namely, since the Young's modulus of the fibres $E_{f}$ is greater than that of the matrix $E_{m}$, the penalization weight in the matrix is by a factor of $\left(E_{m} / E_{f}\right)^{2}$ lower than in the fibres. Therefore the ratio of the regularization length in the matrix with respect to the fibre is equal to $\sqrt{E_{m} / E_{f}}(e . g$., if the regularization length in the fibres is equal to 128 pixels, then that in the matrix is equal to 57 pixels with $E_{m} / E_{f}=0.2$ ). 
The same correlation was run for several ratios and the optical residuals $\Phi_{o}^{2}(1)$ are shown in Fig. 10a. The larger the ratio between the Young's moduli, the smaller the optical residual. Increasing the elastic contrast between the fibres and the matrix with the same regularization length in the fibres implies decreasing the regularization weight in the matrix. Thus, the optical residuals in the matrix decrease (as more freedom is given to the correlation scheme) while they do not increase in the fibres because their strain levels are very low. With the aim of measuring displacement fields within the matrix at the microscopic scale, it is interesting to increase the ratio between these properties since it reduces the difference between the initial and the corrected deformed pictures.

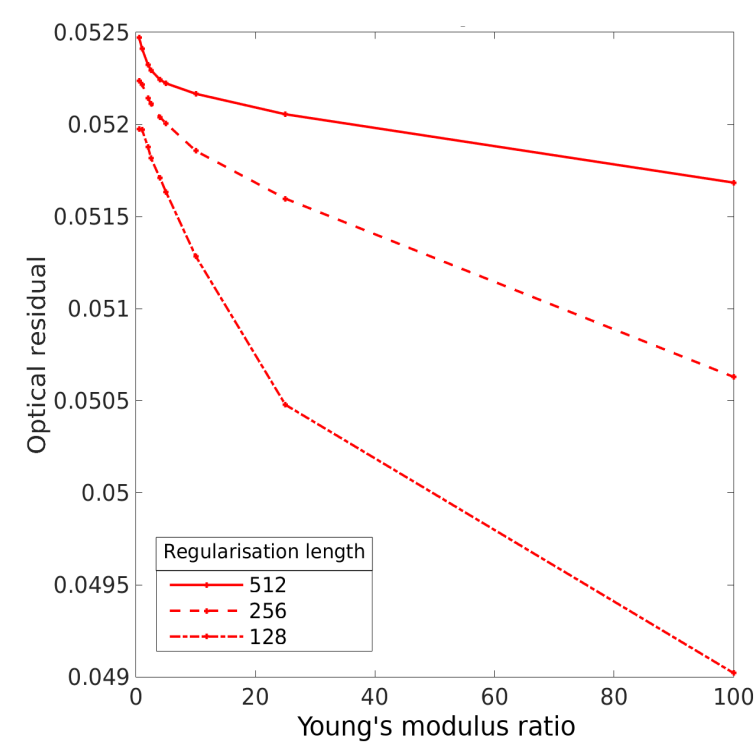

(a)

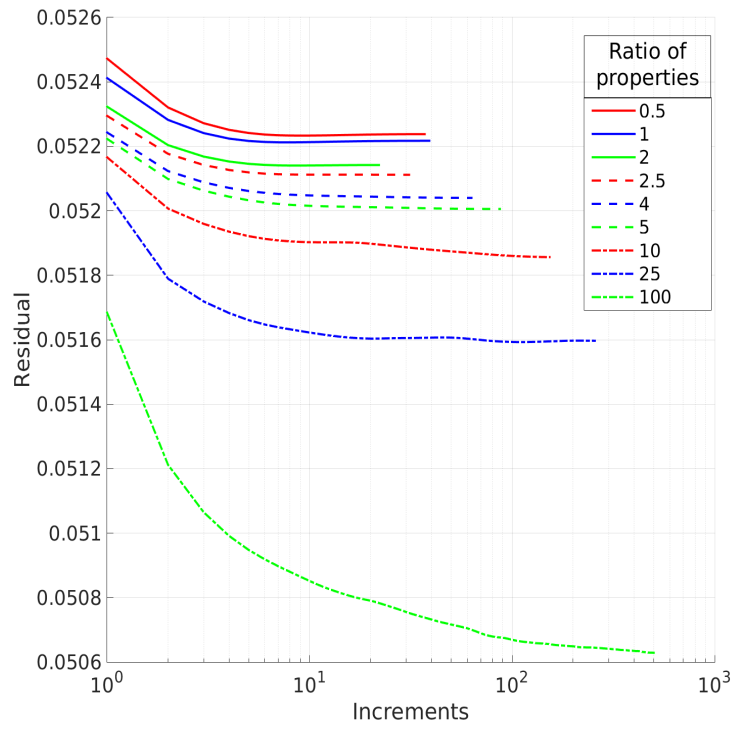

(b)

Fig. 10: (a) Changes in the optical residual as a function of Young's modulus ratios for several regularization lengths. (b) Optical residual at fixed regularization length (i.e., 128 pixels) as a function of the iteration number for several Young's modulus ratios.

However, using a high ratio between the elastic properties has two consequences. First, it gives more freedom for the displacement measured in the matrix. The measurement is then more influenced by noise and the minimization process requires more iterations to converge and thus more time (Fig. 10b). Second, it implies making the assumption that the fibre does not deform much. The aim being to measure the displacements all over the pictures, it may be considered as a strong assumption. However, numerical simulations have shown that the strains in the fibre is really small, and the displacement is barely measurable with the present resolution. The assumption is thus reasonable and using a high ratio of properties can then reduce the correlation residuals. 
If no information is available, using large Young's modulus ratios is a good practice. However, if the elastic properties of fibres and matrix are known, then it is better to take these data and use a physically-based regularization.

\section{Conclusions}

Fibre/matrix debonding was observed through in situ experimental tests in the SEM and a speckle pattern was adapted to improve the measure by digital image correlation. A comparison between local and FE-DIC with mechanical regularization was performed on pictures acquired during the experimental tests. The comparison was done with and without a speckle pattern to choose the most appropriate method to measure displacement fields. It was shown that in the presence of a speckle pattern, both approaches can give valuable results. However, without any speckle pattern, FE-DIC with mechanical regularization can measure displacement fields with a resolution not reached with local DIC.

The first improvement was to create a mesh in agreement with the microstructure of the composite. The use of this mesh has the advantage of preventing the existence of elements lying across the fibre/matrix interface. Thus, the mechanical residuals are localized in the area where there is a real difference between the physical phenomenon (fibre/matrix debonding) and the elastic model used in the mechanical regularization. This improvement offers the possibility of detecting the apparition of debondings and to follow their evolution during the whole mechanical test. The second improvement was to take into account the difference in mechanical properties between fibres and matrix. This improvement allows to take into account the microstructure in the displacement and strain fields. Moreover, it is possible to measure displacement fields in the interfibre matrix with a standard uncertainty of $10 \mathrm{~nm}$ (a fifth of a pixel) without the use of a speckle pattern. Last, because FEDIC uses a mesh to measure displacement fields, the same mesh can be used in mechanical (FE) simulations. Consequently, the displacement field measured during the experimental test can also give the boundary conditions that can be used to validate the numerical model or calibrate material parameters.

\section{Acknowlegments}

This work was funded by Safran Composites. The authors also thank D. Boivin and N. Horezan for all the fruitful discussions and advises concerning the optimal use of the SEM. 


\section{References}

1. Spoltman M W, Bowerman R D, and Edinger R L (March 7 1989). Composite aircraft propeller blade, US Patent $4,810,167$.

2. Daggumati S, De Baere I, Van Paepegem W, Degrieck J, Xu J, Lomov S V, and Verpoest I (2010) Local damage in a 5-harness satin weave composite under static tension: Part I-experimental analysis. Composites Science and Technology, 70(13):1926-1933.

3. Gao F, Boniface L, Ogin S L, Smith P A, and Greaves R P (1999) Damage accumulation in woven-fabric CFRP laminates under tensile loading: Part 1. observations of damage accumulation. Composites Science and Technology, 59(1):123-136.

4. Ivanov D S, Baudry F, Van Den Broucke B, Lomov S V, Xie H, and Verpoest I (2009) Failure analysis of triaxial braided composite. Composites Science and Technology, 69(9):1372-1380.

5. Osada T, Nakai A, and Hamada H (2003) Initial fracture behavior of satin woven fabric composites. Composite structures, 61(4):333-339.

6. Yang L, Yan Y, Liu Y, and Ran Z (2012) Microscopic failure mechanisms of fiber-reinforced polymer composites under transverse tension and compression. Composites Science and Technology, 72(15):18181825.https://www.overleaf.com/project/5c4c93511913523b26fab11a

7. Lisle T, Bouvet C, Pastor M L, Rouault T, and Margueres P (2015) Damage of woven composite under tensile and shear stress using infrared thermography and micrographic cuts. Journal of materials science, 50(18):61546170 .

8. Doitrand A, Fagiano C, Chiaruttini V, Leroy F H, Mavel A, and Hirsekorn M (2015) Experimental characterization and numerical modeling of damage at the mesoscopic scale of woven polymer matrix composites under quasi-static tensile loading. Composites Science and Technology, 119:1-11.

9. Koimtzoglou C, Dassios K G, and Galiotis C (2009) Effect of fatigue on the interface integrity of unidirectional $\mathrm{C}_{\mathrm{f}}$-reinforced epoxy resin composites. Acta Materialia, 57(9):2800-2811.

10. Herrera-Franco P J and Drzal L T (1992) Comparison of methods for the measurement of fibre/matrix adhesion in composites. Composites, 23(1):2-27.

11. Lecomte-Grosbras P, Palush B, Brieu M, De Saxcé G, Sabatier L (2009) Interlaminar shear strain measurement on angle-ply laminate free edge using digital image correlation. Composites Part A: Applied Science and Manufacturing, 40(12):1911-1920

12. Leong M, Overgaard L C T, Thomsen O T, Lund E, Daniel I M (2012) Investigation of failure mechanisms in GFRP sandwich structures with face sheet wrinkle defects used for wind turbine blades. Composite Structures, 94(2):768-778.

13. Fitoussi J, Meraghni F, Jendli Z, Hug G, Baptiste D (2005) Experimental methodology for high strain-rates tensile behaviour analysis of polymer matrix composites. Composites Science and Technology, 65(14):21742188.

14. Canal L P, González C, Molina-Aldareguía J M, Segurado J, and LLorca J (2012) Application of digital image correlation at the microscale in fiber-reinforced composites. Composites Part A: Applied Science and Manufacturing, 43(10):1630-1638.

15. Mortell D J, Tanner D A, and McCarthy C T (2014) In-situ SEM study of transverse cracking and delamination in laminated composite materials. Composites Science and Technology, 105:118-126.

16. O'Dwyer D J, O'Dowd N P, and McCarthy C T (2014) In-situ SEM mechanical testing of miniature bonded joints. International Journal of Adhesion and Adhesives, 50:57-64.

17. Sutton M A, Wolters W J, Peters W H, Ranson W F, and McNeill S R (1983) Determination of displacements using an improved digital correlation method. Image and vision computing, 1(3):133-139. 
18. Hild F and Roux S (2006) Digital image correlation: from displacement measurement to identification of elastic properties-a review. Strain, 42(2):69-80.

19. Pan B, Qian K, Xie H, and Asundi A (2009) Two-dimensional digital image correlation for in-plane displacement and strain measurement: a review. Measurement science and technology, 20(6):062001.

20. Kammers A D and Daly S (2013) Digital image correlation under scanning electron microscopy: methodology and validation. Experimental Mechanics, 53(9):1743-1761.

21. David C (2014) Passieux J C, Bugarin F, David C, Périé, J N, and Robert L (2015) Multiscale displacement field measurement using digital image correlation: Application to the identification of elastic properties. Experimental Mechanics, 55(1):121-137.

22. Brillaud J and Lagattu F (2002) Limits and possibilities of laser speckle and white-light image-correlation methods: theory and experiments. Applied Optics, 41(31):6603-6613.

23. Mehdikhani M, Aravand M, Sabuncuoglu B, Callens M G, Lomov S V, and Gorbatikh L (2016) Full-field strain measurements at the micro-scale in fiber-reinforced composites using digital image correlation. Composite Structures, 140:192-201.

24. Li N, Guo S, and Sutton M A (2011) Recent progress in e-beam lithography for sem patterning. In $M E M S$ and Nanotechnology, Volume 2, pages 163-166. Springer.

25. Qin D, Xia Y, and Whitesides G M (2010) Soft lithography for micro-and nanoscale patterning. Nature protocols, 5(3):491-502.

26. Tanaka Y, Naito K, Kishimoto S, and Kagawa Y (2011) Development of a pattern to measure multiscale deformation and strain distribution via in situ FE-SEM observations. Nanotechnology, 22(11):115704.

27. Collette S A, Sutton M A, Miney P, Reynolds A P, Li X, Colavita P E, Scrivens W A, Luo Y, Sudarshan T, Muzykov P, et al (2004) Development of patterns for nanoscale strain measurements: I. fabrication of imprinted $\mathrm{Au}$ webs for polymeric materials. Nanotechnology, 15(12):1812-1817.

28. Wang H, Xie H, Li Y, and Zhu J (2012) Fabrication of micro-scale speckle pattern and its applications for deformation measurement. Measurement Science and Technology, 23(3):035402.

29. Berfield T A, Patel J K, Shimmin R G, Braun P V, Lambros J, and Sottos N R (2007) Micro-and nanoscale deformation measurement of surface and internal planes via digital image correlation. Experimental Mechanics, $47(1): 51-62$.

30. Sutton M A, Mingqi C, Peters W H, Chao Y J, and McNeill S R (1986) Application of an optimized digital correlation method to planar deformation analysis. Image and Vision Computing, 4(3):143-150.

31. Chevalier L, Calloch S, Hild F, and Marco Y (2001) Digital image correlation used to analyze the multiaxial behavior of rubber-like materials. European Journal of Mechanics, 20:169-187.

32. Sun Y, Pang J H L, Wong C K, and Su F (2005) Finite element formulation for a digital image correlation method. Applied Optics, 44(34):7357-7363.

33. Besnard G, Hild F, and Roux S (2006) "Finite-element" displacement fields analysis from digital images: application to Portevin-Le Châtelier bands. Experimental Mechanics, 46(6):789-803.

34. Mortazavi F (2013) Development of a global digital image correlation approach for fast high-resolution displacement measurements. Doctoral dissertation, École Polytechnique de Montréal.

35. Réthoré J, Hild F and Roux S (2008) Extended digital image correlation with crack shape optimization. International Journal for Numerical Methods in Engineering, 73(2):248-272.

36. Passieux J C, Périé J N (2012) High resolution digital image correlation using proper generalized decomposition: PGD-DIC. International Journal for Numerical Methods in Engineering,92(6):531-550.

37. Réthoré J, Roux S, and Hild F (2009) An extended and integrated digital image correlation technique applied to the analysis of fractured samples: The equilibrium gap method as a mechanical filter. European Journal of Computational Mechanics/Revue Européenne de Mécanique Numérique, 18(3-4):285-306. 
38. Tomičević Z, Hild F, and Roux S (2013) Mechanics-aided digital image correlation. The Journal of Strain Analysis for Engineering Design, 48:330-343.

39. Hild F and Roux S (2012) Comparison of local and global approaches to digital image correlation. Experimental Mechanics, 52(9):1503-1519.

40. Correlated Solutions (2009) Vic-2d. Reference Manual.

41. Réthoré J (2010). A fully integrated noise robust strategy for the identification of constitutive laws from digital images. International Journal for Numerical Methods in Engineering, 84(6):631-660.

42. Sutton M A (2013) Computer vision-based, noncontacting deformation measurements in mechanics: a generational transformation. Applied Mechanics Reviews, 65(5):1-23.

43. Bonnet M and Constantinescu A (2005) Inverse problems in elasticity. Inverse problems, 21(2):R1.

44. Zhou P and Goodson K E (2001) Subpixel displacement and deformation gradient measurement using digital image/speckle correlation (disc). Optical Engineering, 40(8):1613-1620.

45. Asp L E (1998) The effects of moisture and temperature on the interlaminar delamination toughness of a carbon/epoxy composite. Composites Science and Technology, 58(6):967-977.

46. Assarar M, Scida D, El Mahi A, Poilâne C, and Ayad R (2011) Influence of water ageing on mechanical properties and damage events of two reinforced composite materials: Flax-fibres and glass-fibres. Materials \& Design, 32(2):788-795.

47. HexcelCorporation (1998) HexFlow® RTM 6 Product Data. ww.hexcel.com /Resources/DataSheets/RTM - Data - Sheets/RTM6_global.pdf.

48. HexcelCorporation (1998) HexTow $\mathbb{R}$ AS7 Product Data. ww.hexcel.com /user_area/content_media/raw/AS7_Industrial_HexTow_DataSheet.pdf.

49. Maurin R, Davies P, Baral N, Baley C (2008) Transverse properties of carbon fibres by nano-indentation and micro-mechanics. Applied Composite Materials, 15(2):61-73.

50. Herráez M, Fernández A, Lopes C S, González C (2016) Strength and toughness of structural fibres for composite material reinforcement. Phil. Trans. R. Soc. A, 374(2071):1-11.

51. Morelle X P, Chevalier J, Bailly C, Pardoen T, Lani F (2017) Mechanical characterization and modeling of the deformation and failure of the highly crosslinked RTM6 epoxy resin. Mechanics of Time-Dependent Materials, 21:419-454.

52. Sutton M A, Li N, Joy D C, Reynolds, A P, Li X (2007) Scanning Electron Microscopy for Quantitative Small and Large Deformation Measurements Part I: SEM Imaging at Magnifications from 200 to 10,000. Experimental Mechanics, 47(6):775-787.

53. Guery A, Latourte F, Hild F, and Roux S (2013) Characterization of SEM speckle pattern marking and imaging distortion by digital image correlation. Measurement Science and Technology, 25(1):015401.

54. Sutton M A, Li N, Garcia D, Cornille N, Orteu J J, McNeill S R, Schreier H W, Li X (2006) Metrology in a scanning electron microscope: theoretical developments and experimental validation. Measurement Science and Technology, 17(10):2613-2622. 